\title{
Efficient rendering of caustics with streamed photon mapping
}

\author{
K. GUZEK and P. NAPIERALSKI* \\ Institute of Information Technology, Lodz University of Technology, 215 Wolczanska St., 90-924 Lodz, Poland
}

\begin{abstract}
In this paper, we present the streamed photon mapping method for enhancing the rendering of caustics. In order to achieve a realistic caustic effect, global illumination methods require additional data, which are gathered by creating a caustic map or increasing the number of samples used for rendering. Our method employs a stream of photons with a varying luminance level depending on the material properties of the surface. The application of a concentrated photon stream provides the ability to render caustics effectively without increasing the number of photons in a photon map. Such an approach increases visibility of results, while also allowing for faster computations.
\end{abstract}

Key words: rendering, global illumination, photon mapping, caustics.

\section{Introduction}

The interaction of light with matter in the real world results in a variety of optical phenomena. Understanding how those phenomena occur and where to implement them is crucial for creating realistic image renders. When observing the reflection or refraction of light through curved surfaces, one may notice some characteristic patches of light, referred to as caustics. The caustic effects can be classified into two types: diacaustics and catacaustics. The former refer to the caustics formed by the refraction of light rays, while the latter denote the caustics formed by the reflection of light rays (Fig. 1). The shape of a caustic is determined by the shape of the reflective or refractive surface, as well as its position in relation to the light source [1].
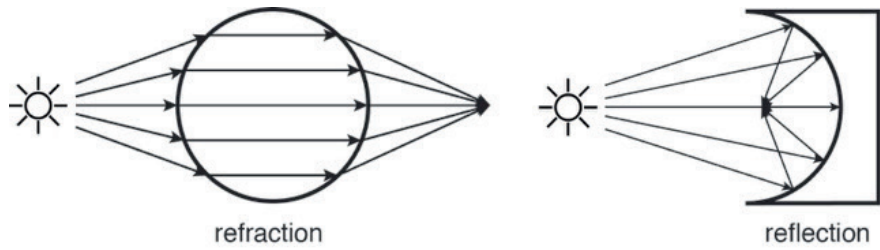

Fig. 1. Scheme showing the generation of diacaustics (on the left) and catacaustics (on the right)

The rendering of caustics involves the simulation of a light path in a virtual scene. In this case the light path can be described by the light transport notation as L(D)*(S)+DE [2]., where $\mathrm{L}$ denotes the light source, $\mathrm{S}$ denotes a specular surface, $\mathrm{D}$ - diffusive surface and $\mathrm{E}-$ the observer. The * operator denotes the occurrence of zero or more events, while the + operator denotes one or more events. However, the simulation of a light transport path is a very complex and computationally expensive process. Thus, the stochastic Monte Carlo methods have been applied to this purpose [3].

\footnotetext{
*e-mail: piotr.napieralski@p.lodz.pl
}

\section{Rendering caustics}

The first attempt to simulate a natural caustic effect was the path tracing method, introduced by James Kajiya in 1986 [4].

However, the method proved to be highly inefficient. The caustics were poorly rendered, as the light source was obscure. A significant improvement was introduced both and independently by Lafortune et al. in 1993 [5] and by Veach et al. in 1994 [6]. They proposed the bi-directional path tracing method. This solution improved the quality of caustics, but the changes in the direction of light reflection resulted in large variance, which in turn increased the amount of noise.

The metropolis light transport method significantly improved the quality of rendered images by decreasing the amount of noise whilst maintaining the crucial attributes associated with global illumination. The use of caustic perturbations provided the ability to achieve a more realistic caustic effect [7].

In 1996, the method based on path tracing was extended by introducing a photon map [8]. This approach was designed to enhance the rendering of both direct and indirect light effects, including caustics. Today, the photon mapping method is one of the most popular algorithms employed by commercial rendering engines, such as V-Ray Chaos Group, Nvidia Mental Ray or YafaRay.

2.1. Rendering caustics with photon mapping. The photon mapping algorithm assumes a two-stage process. The first stage consists in emitting from the light source and tracing through the scene a defined number of photons. When the photon hits the diffusive surface, information about the position of the hit point, photon energy and incoming vector, are saved into a data structure called the photon map. If the surface is specular or refractive, the photon is propagated further according to the law of reflection and Snell's law, until it finally hits the diffusive surface. Then, depending on its coefficient of probability, the photon is either absorbed or reflected with a scaled energy value. The process is repeated until it reaches a specified depth of recursion. The second stage is the classical ray-tracing ren- 
dering of the scene using the information collected in the photon map [9]. Photon mapping estimates the irradiance at a point where a photon strikes, i.e. intersects a surface, by adding up the flux of the nearest photons in the area around that intersection point. The result of irradiance estimation is prone to bias errors. In particular, it may be affected by proximity, boundary or topological bias. The proximity bias is related to the problem of density estimation, relying on the nearest-neighbour density estimation, due to which the estimation result converges with the weighted average of light energy deposited in the area surrounding the intersection point. The boundary bias manifests itself in the darkening of polygon edges, where no photons are stored. Topological bias results from the assumption that the considered geometry is flat. Thus, any curved surface leads to overestimation of flux density [10].

A few methods have been proposed to eliminate the estimation bias. Ray mapping, proposed by Lastra et al. [11], departed from the assumption that the area surrounding the sampling point is flat. In their method, the density estimation is performed on the plane tangent to a surface point on the basis of the vector of photon propagation direction rather than the very hit point. An example of an efficient ray map was introduced by Havran [12]. Tobler and Maierhofer [13] suggested replacing the circular estimation area with an octagon or eight octagons to eliminate undesired shadow leaks close to wall corners. Such an approach enables precise estimation of indirect illumination close to the edges of objects and on relatively small isolated objects.

Frisvad et al. [14] proposed a photon splatting technique which reduces noise and blur in the rendering of caustics. They used photon differentials to determine the size and shape of the splats such that we achieve adaptive anisotropic flux density estimation in photon splatting. Roland Schregle [15] proposed a bias compensating operator for nearest-neighbour density estimation which adapts the bandwidth according to the estimated bias in the reconstructed illumination.

Hachisuka et al. [16] developed an extension to photon mapping - progressive photon mapping, which uses a new radiance estimate that approximates the radiance value as more photons are used.

A method which enables a more accurate density estimation is grouped photon mapping [17]. It extends the first step of Jensen's method by adding a set of operations aimed at clustering the photons deposited in a photon map into groups. The photons are grouped according to the direction of their propagation in a scene. Thus, the photon map, structured in the form of a k-d tree, contains photon groups instead of individual photons. Those groups are in turn used to create polygons, instead of the standard circular areas, for a more accurate estimation of photon energy density.

Another problem related to irradiance estimation is the clarity of results. It is extremely important to adapt the photon settings to the scene's geometry and materials involved. A non-uniform radiance distribution can have a significant impact on the final caustic effect. There is a considerable number of methods that employ additional caustic maps to enhance the rendering of caustics. Such an approach requires storing large amounts of data. Another crucial element of methods based on photon mapping is the selection of the data search technique applied to radiance estimation. It is assumed that most (95\%) of the rendering time is used for gathering photons [18].

Rescaling photon energy could affect the simulation of light concentration, while preserving single data structure.

\section{Enhancing caustics with streamed photon mapping}

The streamed photon mapping method proposed in [19] is a modification of the classic photon mapping. Its aim is to enhance the search for neighbouring photons by assembling photons into streams and thus improve the quality of global illumination effects, such as caustics, colour bleeding and soft shadows.

A stream of photons is defined by the so called leading photon, which indicates the direction of propagation of the stream, and a number of associated photons $\left(n_{f a}\right)$, with directions of propagation deviating from the direction of the leading photon in the area with a radius $\left(r_{s}\right)$. The number of streams $\left(n_{f s}\right)$, the number of associated photons in the streams $\left(n_{f a}\right)$ and radius $\left(r_{s}\right)$ are parameters defined by the user. The input energy of a single stream is calculated based on the energy of the light source and the number of emitted streams, according to the following formula:

$$
E_{s}=\frac{E_{z}}{n_{f s}},
$$

where:

$E_{s}$ - energy of a single stream of photons,

$E_{z}$ - energy of the source of light,

$n_{f s}$ - number of the streams of photons emitted by the source.

The energy of the stream is then equally divided among all the photons in the stream $\left(n_{f a+1}\right)$. The number of associated photons in particular streams, and thus the energy of those streams, can be reduced during their propagation in the scene.

3.1. Stream propagation algorithm. The propagation of photon streams in the scene is performed according to the following algorithm (Fig. 2):

1. Determine a new direction of the leading photon:

a) when the leading photon hits a diffusive surface area ( $\mathrm{Fa}$ and $\mathrm{Fb}$ ), it bounces off in a stochastically determined direction within the hemisphere around the hit point,

b) when the leading photon hits a specular surface (Fa'), it bounces in a direction determined in accordance with the law of reflection,

c) litem when the leading photon strikes a refractive surface $\left(\mathrm{Fb}^{\prime}\right)$, the direction of its refraction is determined in accordance with Snell's law.

2. Test the intersections of the ray designated by the photon's direction vector with the geometry of the scene:

a) if no intersection is found, the propagation of the stream comes to an end,

b) if an intersection is found, we proceed to Step 3 . 
3. Identify the type of material assigned to the given intersection point:

a) in the case of a diffusive surface, each associated photon is assigned a random point within the region surrounding the new position of the leading photon of a radius denoting the stream's width ( $\mathrm{Fa}$ and $\mathrm{Fb}$ ). The new direction of propagation of each associated photon is determined considering the stream decay mechanism [18]. When the propagation process of all associated photons in the stream is complete, we check if the maximum permitted number of bounces ( $\mathrm{mr}$ ) of the leading photon has been achieved:

- if not - we go back to step 1;

- if yes - the propagation of the stream comes to an end.

b) in the case of a specular or refractive surface, all associated photons are eliminated and the sum of their energy values is assigned to the leading photon ( $\mathrm{Fa}^{\prime}$ and $\left.\mathrm{Fb}^{\prime}\right)$. Then, we go back to Step 1.

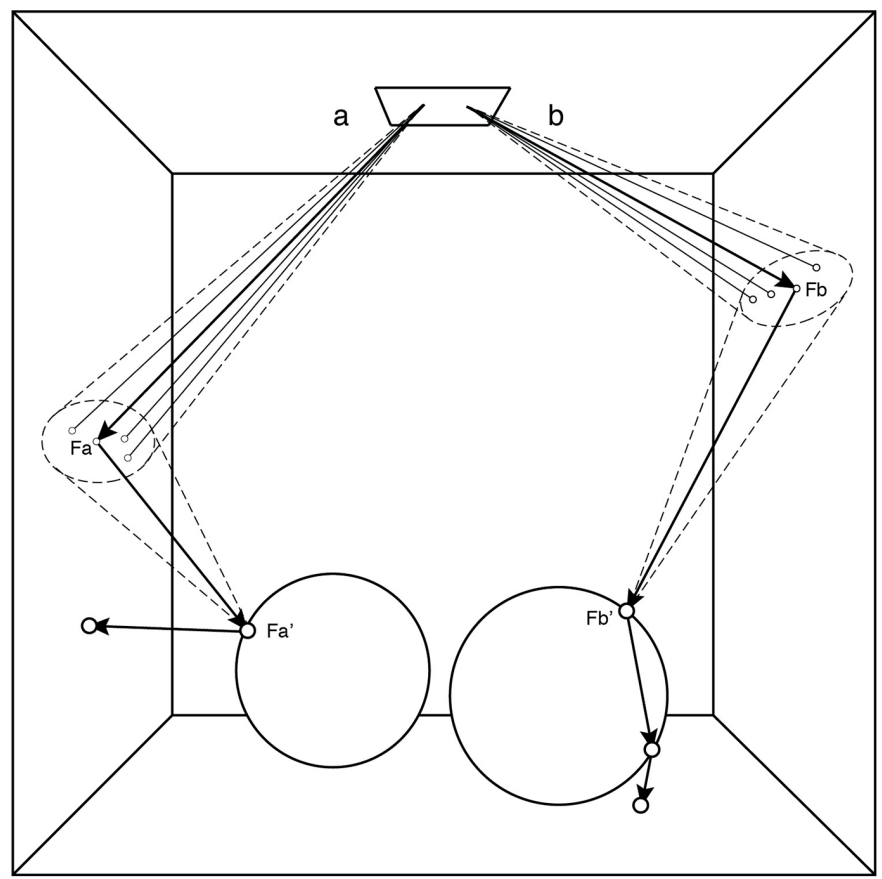

Fig. 2. Propagation of photon streams for different types of surface: diffuse $(\mathrm{Fa}$ and $\mathrm{Fb})$, specular $\left(\mathrm{Fa}^{\prime}\right)$, refractive $\left(\mathrm{Fb}^{\prime}\right)$

The behaviour of the leading photon which undergoes refraction or reflection, regardless of the radius of the stream and the number of associated photons, is implemented in such a way as to stop further stochastic distribution of associated photons and thus eliminate them from the stream, which prevents the distortion of caustic effects.

The above stream propagation algorithm increases the concentration of the energy of photon streams as a result of reflection and refraction processes, with the aim of achieving a more visible caustic effect. The maximum permitted number of bounces (mr) of the leading photon from the diffusive surface is a parameter set by the user.
3.2. Rendering streams of photons. Each bounce of the leading photon from the diffusive surface is recorded in the $\mathrm{k}-\mathrm{d}$ tree, together with the information about the associated photons. Thus, a map of photon streams is created, which stores information about the distribution of photon hit points on the geometry of the scene, the energy of the photons and the normalised vectors of photon arrival directions in every stream.

Rendering is performed in a manner analogous to that employed in the classic photon mapping. A modified recursive ray tracing method is applied. The search for nearest neighbours is limited to finding the leading photons, each with an array of associated photons which constitute a given stream. Thus, the total number of comparisons needed to perform the search is reduced as compared with the classic photon mapping method. The energy of a given stream is the sum of the energies of the constituent photons.

The radiance per a given hit point $\mathrm{x}$ is calculated on the basis of the sum of energies of the neighbouring streams according to the following formula:

$$
L\left(x, \omega_{r}\right)=\frac{1}{\Delta A} \sum_{i=1}^{k} f_{r}\left(x, \omega_{i}, \omega_{r}\right) E_{i}
$$

where:

$L$ - radiance of the light reflected at point $x$ in the direction $\omega_{r}$, $E_{i}$ - energy of stream $i$,

$f_{r}$ - BRDF (bidirectional reflectance distribution function), similar to that employed in the classic photon mapping,

$\Delta A$ - surface of the circle with a radius equal to radius $R$ of the nearest leading photons search sphere,

$k$ - number of the leading photons found.

\section{Choice of parameters}

For an objective evaluation of image quality, one may apply reference metrics, which require the use of a correct image pattern [20-23]. In many cases of photorealistic image quality evaluation, such a reference image does not exist. The assessment metrics such as peak signal-to-noise ratio (PSNR) are not reliable when applied to images rendered using photon mapping. This results form the fact that pixel colour values are calculated stochastically, which inevitably leads to noise generation.

A subjective judgement of image quality is based on the perception and visual experience of the observer. The key features that determine the assessment are: a clear colour bleeding effect caused by the reflection of coloured light from the walls, gradient smoothness, no redundant spots (noise), smooth transitions between brightness levels and faithful representation of optical phenomena, such as caustics.

The present study involved perceptual tests which were designed and conducted to enable the evaluation of the influence of particular parameters of the SPM method on the quality of images. A total of 60 persons (18-24 years old) were examined. They were divided into two groups: naive respondents and specialists in the field of image synthesis. The respondents compared the images rendered against the reference patterns 
and evaluated them in terms of noise and colour spots using a five-level Likert scale.

The experiment involved a set of test scenes based on the Cornell box model for diffuse, specular and refractive surfaces, taking into account the three-dimensional space of the following parameters (Fig. 3):

- number of photon streams

- number of associated photons

- stream radius

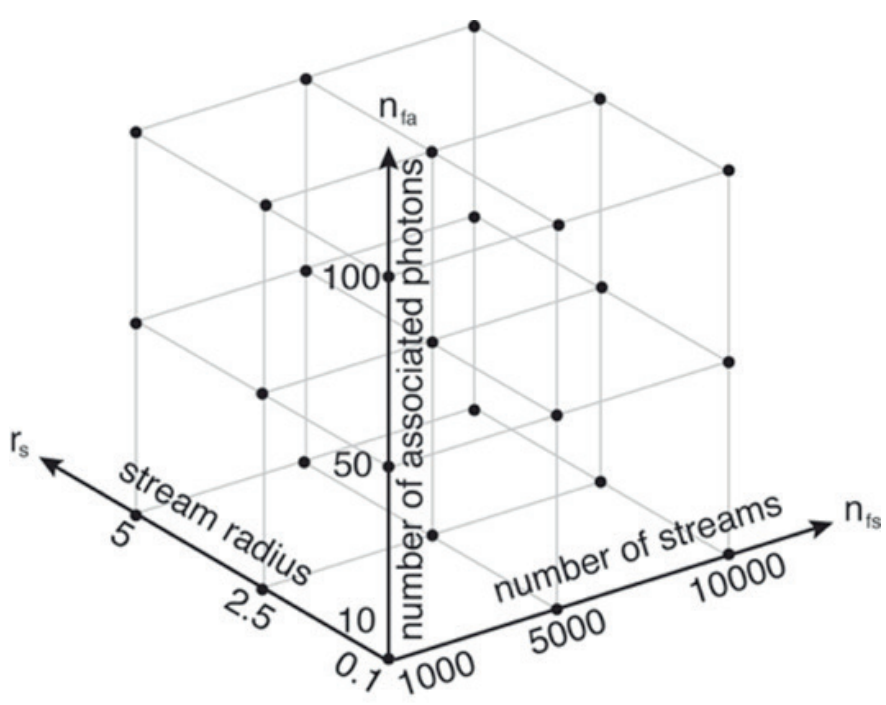

Fig. 3. The space of the SPM parameters tested

The choice of parameter values was made while maintaining fixed rendering parameters of the scene: the radius of nearest-neighbour search area equal to 3 and the number of nearest streams to be found equal to 100 . The maximum number of stream bounces permitted was equal to 4 . The value of stream radius was determined on the basis of the test scene's dimensions $(20 \times 20 \times 20$ scene units $)$. With a radius greater than 5 ,

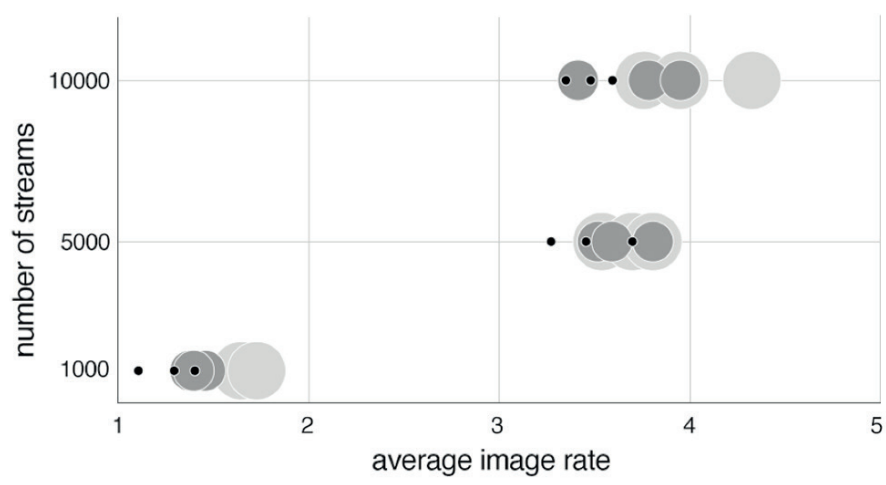

radius

$$
\text { - } r=0.1 \bigcirc r=2.5
$$$$
r=5
$$

Fig. 4. The influence of the number of streams emitted on the average image assessment rate. The larger the radius of a photon stream, the higher the average assessment rate for a given set of images tested the stream's width exceeded half the width of the scene, which led to a significant amount of noise, since the associated photons were too widely scattered.

A number of streams below 1,000 caused major artifacts in the form of colour spots, since the number of photons propagated in the scene was insufficient. With a number of streams above 10,000 and a number of associated photons in a stream above 100, no significant improvement was observed while maintaining the same fixed rendering values.

The greater the number of photons emitted, the higher the average assessment rate (Table 1, Fig. 4). However, the growing

Table 1

Average assessment rates of images generated using the SPM method depending on the parameters used

\begin{tabular}{|c|c|c|c|c|c|c|c|c|}
\hline 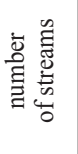 & 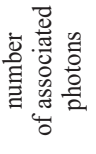 & 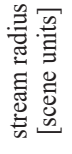 & 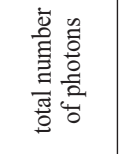 & 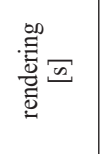 & 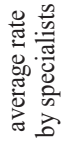 & 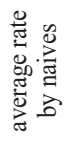 & 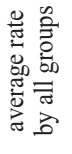 & 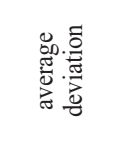 \\
\hline 1,000 & 10 & 0.1 & 10,000 & 109.145 & 1.111 & 1.105 & 1.108 & 0.2285318 \\
\hline 1,000 & 10 & 2.5 & 10,000 & 112.417 & 1.389 & 1.368 & 1.379 & 0.5041551 \\
\hline 1,000 & 10 & 5 & 10,000 & 110.528 & 1.556 & 1.789 & 1.673 & 0.6578947 \\
\hline 1,000 & 50 & 0.1 & 50,000 & 140.147 & 1.222 & 1.368 & 1.295 & 0.4418282 \\
\hline 1,000 & 50 & 2.5 & 50,000 & 127.024 & 1.389 & 1.526 & 1.458 & 0.5415512 \\
\hline 1,000 & 50 & 5 & 50,000 & 123.766 & 1.556 & 1.737 & 1.646 & 0.5983379 \\
\hline 1,000 & 100 & 0.1 & 100,000 & 162.173 & 1.333 & 1.474 & 1.404 & 0.5401662 \\
\hline 1,000 & 100 & 2.5 & 100,000 & 153.187 & 1.111 & 1.684 & 1.398 & 0.5540166 \\
\hline 1,000 & 100 & 5 & 100,000 & 146.197 & 1.667 & 1.789 & 1.728 & 0.6980609 \\
\hline 5,000 & 10 & 0.1 & 50,000 & 135.569 & 3.333 & 3.579 & 3.456 & 0.8642659 \\
\hline 5,000 & 10 & 2.5 & 50,000 & 134.633 & 3.367 & 3.368 & 3.518 & 0.6842105 \\
\hline 5,000 & 10 & 5 & 50,000 & 29.376 & 3.5 & 3.579 & 3.539 & 0.5761772 \\
\hline 5,000 & 50 & 0.1 & 5,000 & 8.443 & 3.611 & 3.789 & 3.7 & 0.8725761 \\
\hline 5,000 & 50 & 2.5 & 250,000 & 176.033 & 3.667 & 3.947 & 3.807 & 0.6565096 \\
\hline 5,000 & 50 & 5 & 250,000 & 169.893 & 3.611 & 3.789 & 3.7 & 0.7493074 \\
\hline 5,000 & 100 & & 500,0 & 58.660 & 3.389 & 3.158 & 3.273 & 0.6883656 \\
\hline 5,000 & 100 & 2.5 & 500,000 & & 3.5 & 3.684 & 3.592 & 0.728 \\
\hline 5,000 & 100 & 5 & 500,000 & 218.416 & 3.667 & 3.947 & 3.807 & 0.4487534 \\
\hline 10,000 & 10 & 0. & 100,000 & 143.087 & 3.278 & 3.684 & 3.481 & 0.6578947 \\
\hline 10,000 & 10 & & 100,000 & 140.008 & 3.833 & 3.737 & 3.785 & 0.7091412 \\
\hline 10,000 & 10 & 5 & 100,000 & 140.765 & 3.833 & 3.684 & 3.759 & 0.7146814 \\
\hline 10,000 & 50 & 0.1 & 500,000 & 200.112 & 3.611 & 3.579 & 3.595 & 0.7894736 \\
\hline 10,000 & 50 & 2. & 500,0 & 187.067 & 4.222 & 3.684 & 3.953 & 0.6703601 \\
\hline 10,000 & 50 & 5 & 500,000 & 179.183 & 4.056 & 3.842 & 3.949 & 0.7008310 \\
\hline 10,000 & 100 & 0.1 & $1,000,000$ & 268.904 & 3.333 & 3.368 & 3.351 & 0.9099722 \\
\hline 10,000 & 100 & 2.5 & $1,000,000$ & 245.609 & 3.722 & 3.105 & 3.414 & 0.7617728 \\
\hline 10,000 & 100 & 5 & $1,000,000$ & 231.808 & 4.389 & 4.263 & 4.326 & 0.7105263 \\
\hline
\end{tabular}


tendency is non-linear and, with more than 250,000 photons emitted, the average image assessment rate is subject to slight changes, taking into consideration the average variation.

All the images generated using 5,000 and 10,000 photon streams obtained an average assessment rate higher than 3 (Table 1). Among the images of the same number of streams emitted, the highest rated ones were mostly those with larger stream radius (Fig. 4).

As demonstrated by test results, for a smaller total number of photons emitted, the images with a larger number of streams and smaller number of associated protons were rated higher than those with a smaller number of streams and higher number of associated photons (Table 1). This results from the fact that, with a small number of streams, too large a number of associated photons introduces a considerable amount of noise into the image. With a total number of photons exceeding 250,000, the average image assessment rate does not vary much depending on the ratio between the number of streams and the number of associated photons.

The tests enabled us to establish an optimal value of radius parameter ( 5 scene units) for considered test scene based on the subjective assessment of the observers.

As a result of the propagation of streams with radius smaller than the dimensions of geometric objects one can observe stronger color bleeding due to the propagation of associated photons in spaces between the objects and the uniform distribution of photons at a relatively small area (Fig. 5).

For scenes with complex geometry (e.g. Stanford bunny) with too broad stream of photons compared to the cavities of the geometry, small errors of shading appear due to excessive scattering of photons in a stream (Fig. 6).

\section{Results}

In order to test the efficiency of our method for caustic generation, we compared the scenes rendered with streamed photon mapping (SPM) against those rendered with the classic photon mapping (PM) [8], taking into account the chosen parameter setting. Both test scenes contained objects which generate diacaustic and catacaustic effects - a glass sphere with an index of refraction $\mathrm{IOR}=1.5$ and a specular surface with a radius of curvature equal to 3.5 scene units, respectively. The scenes were created using the standard Cornell box model (dimensions: $20 \times 20 \times 20$ scene units), as illustrated in Fig. 7. The walls were assigned diffuse reflection properties. As shown in the RGB model, the walls were assigned the following colours: left wall - red $(1,0,0)$, right wall - blue $(0,0,1)$, rear, top and bottom - white $(1,1,1)$. We defined one area light source consisting of 25 point lights distributed evenly on a white (RGB: $1,1,1$ ) surface of the size equal to $3 \times 3$ scene units. The resolution of the rendered images was $512 \times 512$ pixels.

The rendering of the test scenes was performed with the same number of photons: 10,000 streams (with 10 photons in each stream) for SPM and 100,000 photons for the classic PM. The same set of parameters was used for both algorithms: the maximum permitted number of bounces $\mathrm{mr}=4$, the radius of
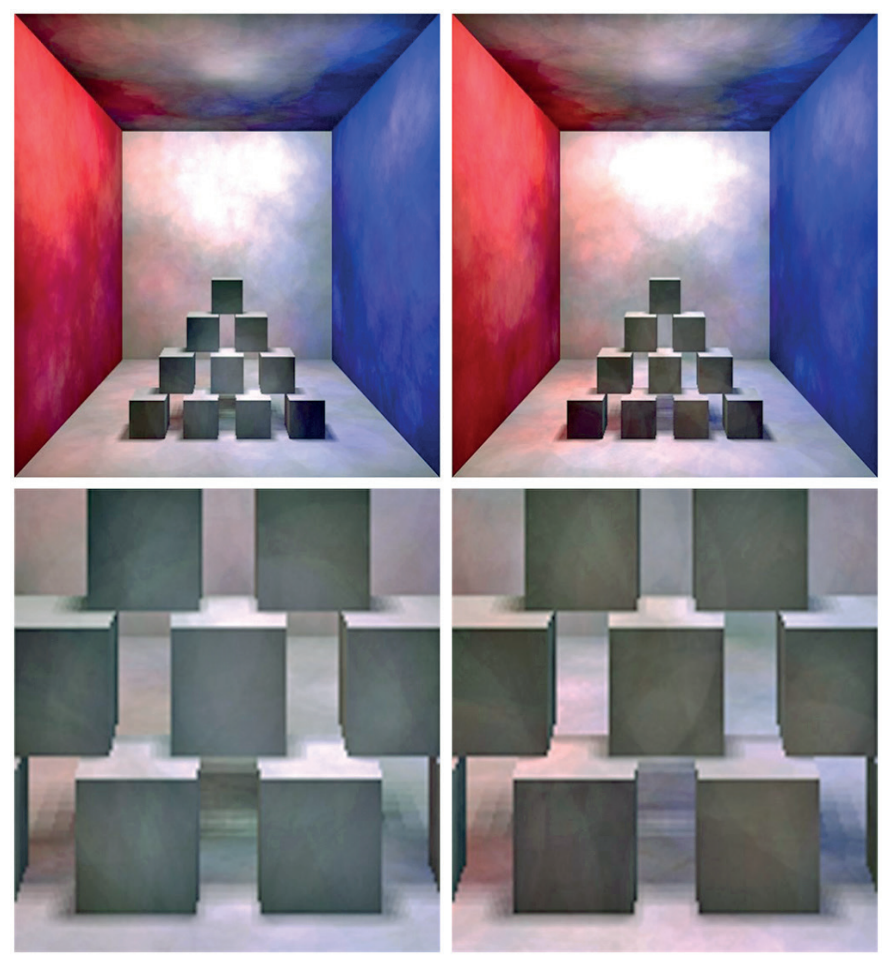

Fig. 5. Test scene with pyramid of boxes rendered using SPM with the following parameters. Left frames: 1,000 streams of photons emitted, 100 associated photons in each stream, stream radius equal to 5 scene units. Right frames: 1,000 streams of photons emitted, 100 associated photons in each stream, stream radius equal to 0.1 scene units

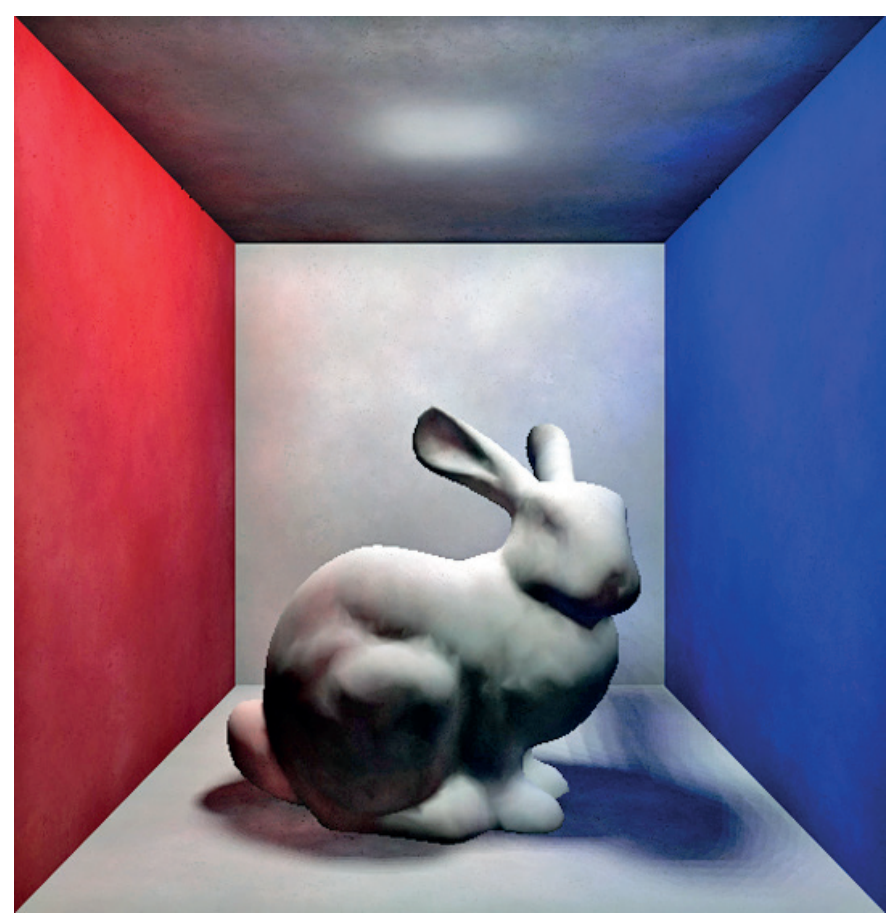

Fig. 6. Test scene with complex geometry (Stanford bunny) rendered using SPM with the following parameters: 10,000 streams of photons emitted, 100 associated photons in each stream, stream radius equal to 2.5 scene units, a maximum of 4 bounces for each stream 


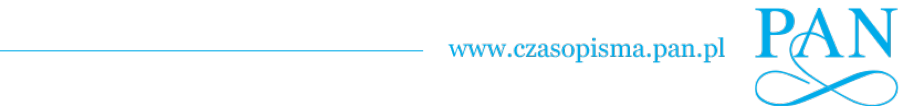

K. Guzek and P." Napieralski
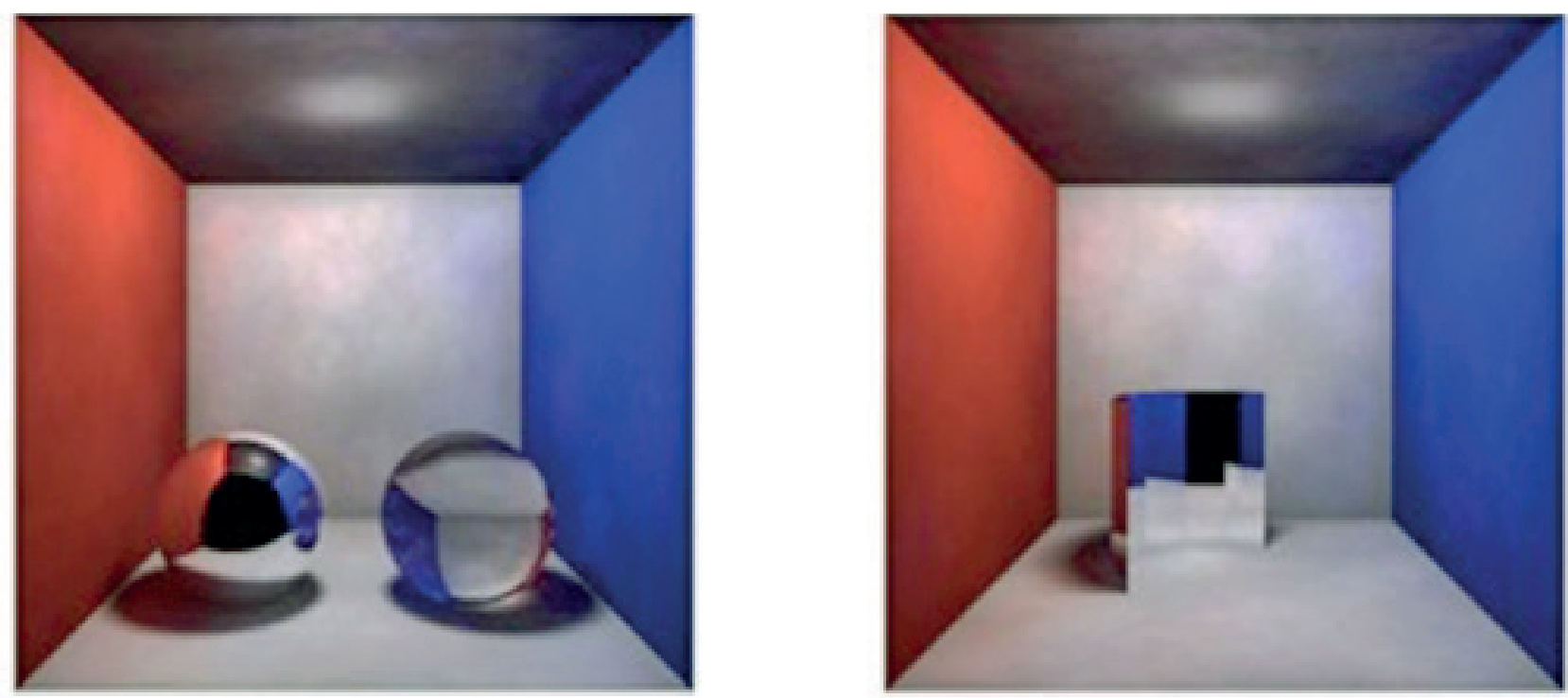

Fig. 7. Upper frame: a diacaustic test scene. Lower frame: a catacaustic test scene. Both scenes were rendered using SPM with the following parameters: 10,000 streams of photons emitted, 10 associated photons in each stream, no additional catacaustic photons emitted, stream radius equal to 5 scene units, a maximum of 4 bounces for each stream
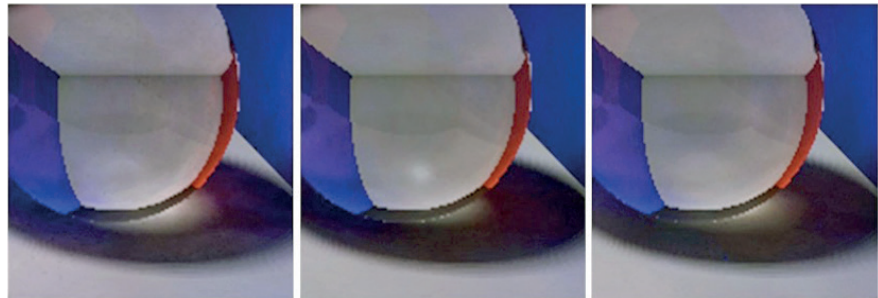

Fig. 8. Left frame: a diacaustic effect achieved by streamed photon mapping using 10,000 streams of 10 photons and radius $r s=5$. Middle frame: a diacaustic effect achieved by photon mapping using 100,000 photons. Right frame: a diacaustic effect achieved by photon mapping using 100,000 global photons and additional 100,000 caustic photons
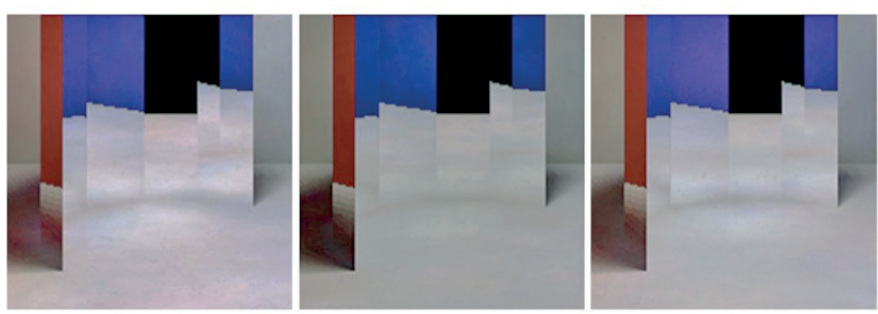

Fig. 9. Left frame: a catacaustic effect achieved by Streamed Photon Mapping using 10000 streams of 10 photons and radius rs $=5$. Middle frame: a catacaustic effect achieved by Photon Mapping using 100000 photons. Right frame: a catacaustic effect achieved by Photon Mapping using 100000 global photons and additional 100000 caustic photons

nearest-neighbour photons search area $\mathrm{R}=3$, the number of nearest neighbours to be found $\mathrm{k}=100$. The SPM provides a better quality of both diacaustics (Fig. 8) and catacaustics (Fig. 9) when compared with the classic PM with no additional emission of caustic photons. To obtain results comparable to SPM, it is necessary for the classic PM method to increase the emission of photons or employ an additional emission of caustic photons (Fig. 8).

The rendering times of particular images are given in Table 2 and Table 3. The application of SPM yields diacaustic and catacaustic effects comparable to those obtained with the classic PM method with an additional emission of caustic photons in a time shorter by $16 \%$ and $10 \%$, respectively.

Table 2

The rendering time and size of the photon map of a diacaustic scene (Fig. 8) depending on the number of photons emitted by a given method

\begin{tabular}{|c|c|c|c|}
\hline method & number of photons & $\begin{array}{c}\text { photon map size } \\
{[\mathrm{MB}]}\end{array}$ & $\begin{array}{c}\text { rendering } \\
{[\mathrm{s}]}\end{array}$ \\
\hline $\begin{array}{l}\text { streamed photon } \\
\text { mapping }\end{array}$ & 10,000 streams $\times$ photons & 7.46 & 90.940 \\
\hline \multirow[b]{2}{*}{ photon mapping } & 100,000 global photons & 7.83 & 95.421 \\
\hline & $\begin{array}{l}100,000 \text { global } \\
+100,000 \text { caustic photons }\end{array}$ & 12.65 & 105.482 \\
\hline
\end{tabular}

Table 3

The rendering time and size of the photon map of a catacaustic scene (Fig. 9) depending on the number of photons emitted by a given method

\begin{tabular}{|c|c|c|c|}
\hline method & number of photons & $\begin{array}{l}\text { photon map size } \\
{[\mathrm{MB}]}\end{array}$ & $\begin{array}{c}\text { rendering } \\
{[\mathrm{s}]}\end{array}$ \\
\hline $\begin{array}{l}\text { streamed photon } \\
\text { mapping }\end{array}$ & $\begin{array}{l}10,000 \text { streams } \\
\times 10 \text { photons }\end{array}$ & 8.29 & 101.103 \\
\hline \multirow[b]{2}{*}{ photon mapping } & 100,000 global photons & 8.35 & 101.794 \\
\hline & $\begin{array}{l}100,000 \text { global } \\
+100,000 \text { caustic photons }\end{array}$ & 13.13 & 111.232 \\
\hline
\end{tabular}




\section{Discussion}

In contrast to the grouped photon mapping method, where the analysis of dependencies between photons, based on their correlated directions, is performed after the photon tracing step [17], our method partitions an ensemble of photons into streams already at the stage of emission and propagation.

The results of rendering show that the streamed photon mapping provides the ability to obtain bright caustic effects thanks to stream concentration in the case reflective or refractive surfaces. It allows one to obtain better results than the classic photon mapping, with no need for additional emission of caustic photons and projection of caustic maps. Another advantage demonstrated by the results is the decreased render time due to splitting an ensemble of photons into streams. The presented method might also include using the algorithm of progressive photon mapping [16] to reduce memory consumption related to photon map storage.

Similar to the classic photon mapping, streamed photon mapping can also apply the kernel density function for a more precise estimation of stream energy density at a given point (e.g. Gauss, Epanechnikov [16]).

Streamed photon mapping proves efficient with relatively large amounts of photons emitted. With too small a number of photons, it produces more artifacts than the classic photon mapping. Streamed photon mapping provides best rendering results when using streams with a width approximately equal to the size of geometric objects involved and the spaces between them. It should be emphasised that for streams with a very small width and a small number of associated photons, the results of streamed photon mapping method become similar to the classic photon mapping.

\section{Conclusion and future work}

In this paper, we have introduced the streamed photon mapping method for rendering caustics, which is an extension of photon mapping developed by Jensen. Our approach provides the ability to obtain a more visible caustic effect when compared with the classic photon mapping. The proposed method requires only one global photon map to render high-quality caustics, whereas most of the existing photon mapping methods require building an additional caustic map, which involves a complex data structure and increases memory requirements. By assembling photons into streams at the very beginning of the propagation process, the method additionally improves the efficiency of the nearest-neighbour search algorithm. As compared to other global illumination rendering methods, the proposed solution enhances significantly the rendering of caustic effects.

The streamed photon mapping method offers a number of options worth pursuing for further development. We believe that this technique might prove practical for the rendering of other global illumination effects, and its optimisation might lead to a significant reduction of the rendering time. There are various directions for future enhancements. For example, the present study has not considered the presence of participating media, such as fog or smoke. The application of the method to dense media seems to be a natural direction for further development. The proposed solution should also be developed in terms of parallel computing to reduce the rendering time.

Acknowledgements. The authors would like to express their gratitude to Prof. Maria Pietruszka for providing invaluable support and counsel at all stages of this research and for lending the expertise essential for the analysis of the research data.

\section{REFERENCES}

[1] M. Pharr and G. Humphreys, Physically Based Rendering: From Theory to Implementation, 2nd ed., Morgan Kaufmann, San Francisco, 2010.

[2] P.S. Heckbert, "Adaptive radiosity textures for bidirectional ray tracing”, ACM SIGGRAPH Computer Graphics 24 (4), 145-154 (1990).

[3] L. Szirmay-Kalos, Monte-Carlo Methods in Global Illumination, VDM, 2000.

[4] J.T. Kajiya, "The rendering equation", ACM SIGGRAPH Computer Graphics 20 (4), ACM, 1986

[5] E.P. Lafortune and Y.D. Willems, "Bi-directional path tracing", Proc. Third International Conference on Computational Graphics and Visualization Techniques (Compugraphics '93), (1993).

[6] E. Veach and L.J. Guibas, "Bidirectional estimators for light transport", 5th Eurographics Workshop on Rendering, 147-162 (1994).

[7] E. Veach and L.J. Guibas, "Metropolis light transport", Proc. Annual Conference on Computer Graphics and Interactive Techniques, SIGGRAPH '97, (1997).

[8] H.W. Jensen, "Global Illumination using photon maps", Proc. Eurographics Workshop on Rendering Techniques '96, (1996).

[9] K. Guzek and P. Napieralski, "Algorithms and methods for global illumination", in Computer Science in Novel Application: Human-Centered Interdisciplinary Applications, pp. 87-102, ed. A. Romanowski, Łódź University of Technology, Łódź, 2012.

[10] R.J. Garcia Hernandez, C. Ureña, J. Poch, and M. Sbert, "Overestimation and underestimation biases in photon mapping with non-constant kernels", IEEE Transactions on Visualization and Computer Graphics 20 (10), 1441-1450 (2014).

[11] M. Lastra, C. Ureña, J. Revelles, and R. Montes, "A particle-path based method for Monte-Carlo density estimation", 13th Eurographics Workshop on Rendering, 33-40 (2002)

[12] V. Havran, J. Bittner, and H.-P. Seidel, "Ray maps for global illumination", Eurographics Symposium on Rendering/Eurographics Workshop on Rendering Techniques, 43-54 (2005).

[13] R.F. Tobler and S. Maierhofer, "Improved illumination estimation for photon maps in architectural scenes", International Conference in Central Europe on Computer Graphics, Visualization, and Computer Vision, 257-262 (2006).

[14] J.R. Frisvad, L. Schjøth, K. Erleben, and J. Sporring, "Photon differential splatting for rendering caustics", Computer Graphics Forum 33 (6), 252-263 (2012).

[15] R. Schregle, "Bias compensation for photon maps", Computer Graphics Forum 22 (4), 729-742 (2003). 
K. Guzek and P. Napieralski

[16] T. Hachisuka, S. Ogaki, and H.W Jensen, "Progressive photon mapping", ACM Transactions on Graphics 27 (5), 130:1-130:8 (2008).

[17] L.-H. Chen, T.-C. Tsai, and Y.-S. Chen, "Grouped photon mapping", The Visual Computer 26 (3), 217-226 (2010).

[18] J. Günther, I. Wald, and P. Slusallek, "Realtime caustics using distributed photon mapping", Proc. 15th Eurographics Symposium on Rendering 2004, 111-121 (2004).

[19] K. Guzek and P. Napieralski, "Streamed Photon Mapping", Metody Informatyki Stosowanej 4/2011 (29), 67-74 (2011).

[20] R.H. Sheikh, A.C. Bovik, and L. Cormack, "No-reference quality assessment using natural scene statistics: JPEG2000", IEEE Trans. on Image Processing 14 (11), 1918-1927 (2005).
[21] W.A. Stokes, J.A. Ferwerda, B. Walter, and D.P. Greenberg, "Perceptual illumination omponents: a new approach to efficient, high quality global illumination rendering", ACM Transactions on Graphics - Proc. ACM Siggraph 200423 (3), 742-749 (2004).

[22] R. Herzog, M. Cadik, T.O. Aydcin, K.I. Kim, K. Myszkowski, and H.-P. Sidel, "NoRM: no-reference image quality metric for realistic image synthesis", Computer Graphics Forum 31 (2), 545-554 (2012).

[23] K. Guzek, and P. Napieralski, "Measurement of noise in the Monte Carlo point sampling method", Bull. Pol. Ac.: Tech. 59 (1), 15-19 (2011).

[24] B.W. Silverman, Density Estimation for Statistics and Data Analysis, CRC Press, London, 1986. 